\title{
The NA60 Experiment: Results and Perspectives
}

\author{
J.M. Heuser*t ${ }^{* \dagger}$ R. Arnaldi**, K. Banicz ${ }^{\ddagger}$, K. Borer ${ }^{\S}$, J. Buytaert $^{\ddagger}$, J. Castor $^{\text {Il }}$, \\ B. Chaurand ${ }^{\|}$, W. Chen ${ }^{\dagger \dagger}$, B. Cheynis ${ }^{\ddagger \ddagger}$, C. Cicalo ${ }^{\S \S}$, A. Colla ${ }^{* *}$, \\ P. Cortese ${ }^{* *}$, A. David"I, A. de Falco ${ }^{\S}$, N. de Marco**, A. Devaux ${ }^{\text {I, }}$
} A. Drees ${ }^{* * *}$, L. Ducroux ${ }^{\ddagger \neq}$, H. En'yo*, A. Ferretti**, M. Floris ${ }^{\S \S}$, P. Force ${ }^{\mathbb{I}}$, A. Grigorian ${ }^{\dagger \dagger \dagger}$, J.-Y. Grossiord ${ }^{\ddagger \ddagger}$, N. Guettet ${ }^{\text {II }}$, A. Guichard ${ }^{\ddagger \ddagger}$, H. Gulkanian ${ }^{\dagger \dagger}$, M. Keil ${ }^{\ddagger}$, L. Klubergll, Z. Li ${ }^{\dagger \dagger}$, C. Lourenço ${ }^{\ddagger}$, J. Lozano ${ }^{\text {IIII }}$, F. Manso ${ }^{\mathbb{I}}$, A. Masoni ${ }^{\S \S}$, A. Neves ${ }^{\text {TI }}$, H. Ohnishi* ${ }^{*}$ C. Oppedisano**, P. Parracho ${ }^{\text {tIT }}$, G. Puddu ${ }^{\S \S}$, E. Radermacher ${ }^{\ddagger}$, P. Rosinsky ${ }^{\ddagger}$, E. Scomparin**, J. Seixas ${ }^{\mathbb{T I}}$, S. Serci ${ }^{\S \S}$, R. Shahoyan ${ }^{\mathbb{T H}}$, P. Sonderegger ${ }^{\mathbb{T I}}$, R. Tieulent ${ }^{\ddagger \ddagger}$, G. Usai ${ }^{\S \S}, H$. Vardanyan ${ }^{\dagger \dagger \dagger}$, R. Veenhof ${ }^{f I I}$ and H. Wöhri ${ }^{\ddagger}$

\author{
${ }^{*}$ RIKEN - The Institute of Physical and Chemical Research, Wako, Saitama, Japan \\ ${ }^{\dagger}$ corresponding author for the NA60 collaboration, e-mail: Johann.Heuser@cern.ch \\ ${ }^{* *}$ University of Turin and INFN, Turin, Italy \\ ${ }^{\ddagger}$ CERN - European Organization for Nuclear Research, Geneva, Switzerland \\ $\S$ University of Bern, Bern, Switzerland \\ ${ }^{4}$ Université Blaise Pascal and CNRS-IN2P3, Clermont-Ferrand, France \\ $\|_{L L R, \text { Ecole Polytechnique and CNRS-IN2P3, Palaiseau, France }}$ \\ ${ }^{\dagger \dagger}$ Brookhaven National Laboratory, Upton, New York, USA \\ ${ }^{\ddagger}$ Université Claude Bernard Lyon-I and CNRS-IN2P2, Lyon, France \\ ${ }^{\$}$ University of Cagliari and INFN, Cagliari, Italy \\ TI Instituto Superior Técnico, Lisbon, Portugal \\ ${ }^{* * *}$ State University of New York at Stony Brook, Stony Brook, New York, USA \\ ${ }^{\dagger \dagger}$ Yerevan Physics Institute, Yerevan, Armenia
}

\begin{abstract}
.
The NA60 experiment takes place at the SPS at CERN, to study the production of open charm and prompt dimuons in collisions induced by proton and heavy ion beams on nuclear targets. For this task, several novel detector systems were added to the dimuon spectrometer and zero-degree calorimeter, which were previously used in the NA50 experiment. The main upgrade is a new silicon pixel vertex spectrometer. It tracks the charged particles that are produced through the collisions in the target and allows us to match their trajectories and momenta with those of the muons that are measured behind the hadron absorber in the muon spectrometer. Besides improving considerably the dimuon mass resolution, the vertex telescope measures the offset of each muon track with respect to the interaction point. This allows us to select events where charm mesons were produced. We present in this article first results from dimuon data that were acquired in Summer 2002 with a $400 \mathrm{GeV}$ proton beam. A silicon microstrip telescope was used at that time, since only a part of the pixel telescope was completed. The results include an improved dimuon mass resolution and an extended phase space coverage when compared with previous experiments. Data from $\mathrm{Pb}-\mathrm{Pb}$ collisions at 20 and $30 \mathrm{GeV}$ per nucleon were collected in October 2002 with three planes of pixel detectors. We show the very good accuracy with which we could reconstruct the interaction vertex. We conclude with a reminder of our physics programme and the ongoing completion of the pixel detector telescope for the Indium-Indium run at $158 \mathrm{GeV}$ per nucleon, scheduled for September and October 2003.
\end{abstract}




\section{PHYSICS MOTIVATION AND OVERVIEW OF THE NA60 EXPERIMENT}

The NA60 experiment [1] at CERN's Super Proton Synchrotron addresses several questions that have been left open by previous experiments of the SPS heavy ion physics programme. NA60 aims at measuring prompt dimuon and open charm production in protonnucleus and nucleus-nucleus collisions. Such measurements have not been performed to date because radiation hard detector technology capable of high-resolution tracking and vertex reconstruction in heavy ion collisions became available only recently. New measurements on open charm production are considered essential to the understanding of intriguing earlier observations. Is the origin of the intermediate mass dimuon excess, observed in the NA38 and NA50 experiments, due to the production of thermal dimuons from a plasma phase? Is the open charm yield enhanced in nucleus-nucleus collisions, and how does this compare with the observed anomalous suppression of charmonium production in central $\mathrm{Pb}-\mathrm{Pb}$ collisions? Which physics variable drives the onset of $\psi^{\prime}$, $\chi_{c}$ and $\mathrm{J} / \psi$ suppression? What is the normal nuclear absorption pattern of the $\chi_{c}$ ?

The concept of the NA60 experiment, taylored towards such measurements, is shown in Fig. 1. A newly instrumented target region complements the muon spectrometer and zero-degree calorimeter that were previously used in the NA50 experiment. A

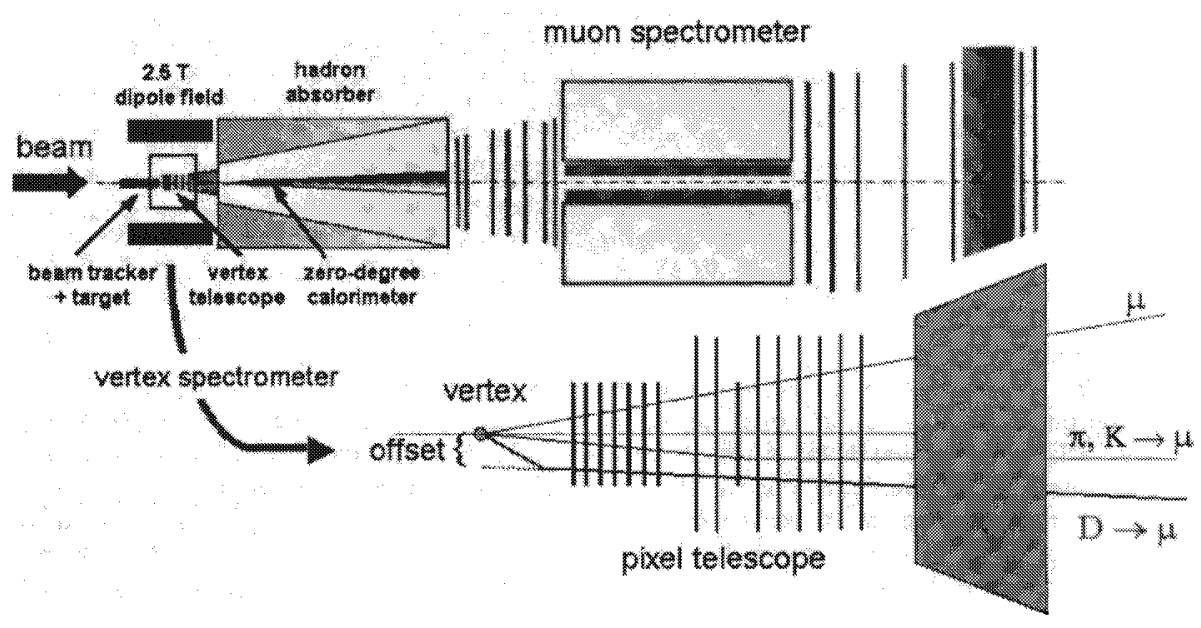

FIGURE 1. Concept of the NA60 experiment. The muon spectrometer and zero-degree calorimeter of NA50 are completed with vertex tracking capabilities for high track densities in heavy ion collisions.

beam tracker upstream and a vertex telescope downstream of the target, in front of the hadron absorber, allow us to determine the interaction point with high precision. The beam tracker [2] comprises two stations of silicon microstrip detectors that are operated at $130 \mathrm{~K}$ to increase its radiation tolerance. It tracks every beam ion and provides both the crossing time and the impact point on the target with good timing and spatial resolution. The vertex spectrometer consists of a multi-plane vertex tracking telescope, shown in Fig. 2, which is built from hybrid silicon pixel detectors that 
were developed at CERN [3]. The telescope is placed inside of a $2.5 \mathrm{~T}$ dipole magnetic field. Its modules are arranged to match the muon spectrometer's angular acceptance of $35<\theta<120 \mathrm{mrad}$. The high granularity pixel detectors are essential to resolve the high track densities in heavy ion collisions. High-resolution track reconstruction and momentum measurement with the vertex spectrometer enable us to match tracks from the muon spectrometer through the hadron absorber into the vertex telescope. The dimuon mass resolution improves considerably, as well as the signal-to-background ratio through a rejection of pion and kaon decays into muons. Furthermore, a precise measurement of muon track offsets makes it possible to separate muons from charm decays or prompt sources.

(a)

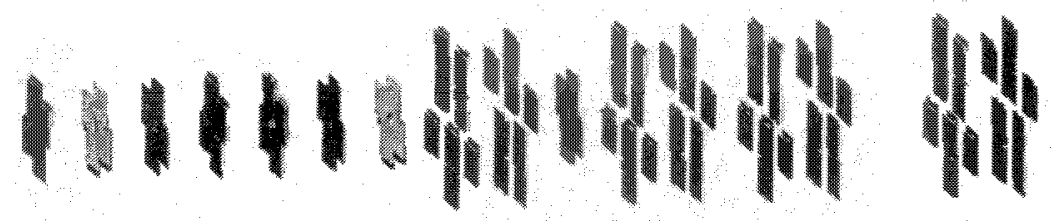

(b)

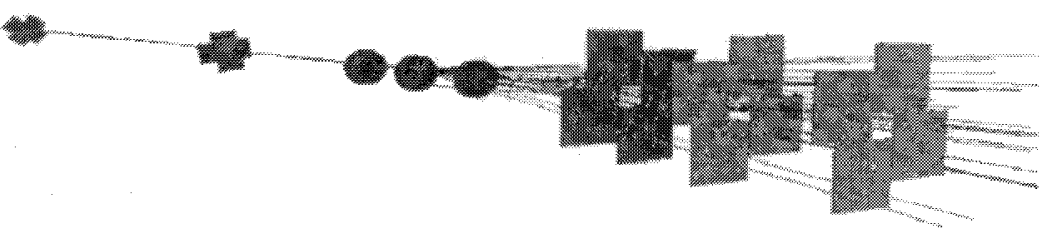

FIGURE 2. (a) Arrangement of pixel detector planes in the vertex spectrometer. Eight 4-chip and eight 8-chip modules are located between $7 \mathrm{~cm}$ and $32 \mathrm{~cm}$ distance from the target. Every hybrid pixel detector chip is segmented into 8192 pixels of $50 \mu \mathrm{m} \times 425 \mu \mathrm{m}$ size.

(b) Operation of the vertex telescope: Average-multiplicity event from a $\mathrm{Pb}-\mathrm{Pb}$ collision at $20 \mathrm{GeV}$ per nucleon in the second target, reconstructed with the beam tracker (seen left from the targets) and three 4-chip pixel detector planes.

\section{FIRST RESULTS FROM PROTON-NUCLEUS COLLISIONS}

The first physics run of NA60 was performed in Summer 2002. Only one pixel detector module could be completed by then. A vertex telescope based on novel microstrip detectors was developed to perform track reconstruction in collisions of $400 \mathrm{GeV}$ protons on six targets made from $\mathrm{Be}$, In and $\mathrm{Pb}$. The telescope comprised eight double-module tracking stations of sensors with variable strip pitch and length. First results from this run are shown in the three panels of Fig. 3. The track reconstruction and vertexing achieved a Z-vertex resolution of $900 \mu \mathrm{m}$, allowing to distinguish collisions in the different targets (Fig. 3a). The dimuon mass measurement was performed with a resolution better than $30 \mathrm{MeV}$ at the $\omega$ and $\phi$ masses. Figure $3 \mathrm{~b}$ shows the result for one of the Be targets. The good mass and vertex resolution will allow us to study with high precision the nuclear dependence of $\omega$ and $\phi$ production. In comparison with previous experiments, the NA60 experiment achieves a much more extended phase space coverage for those measurements, that includes very low transverse momenta (Fig. 3c). 


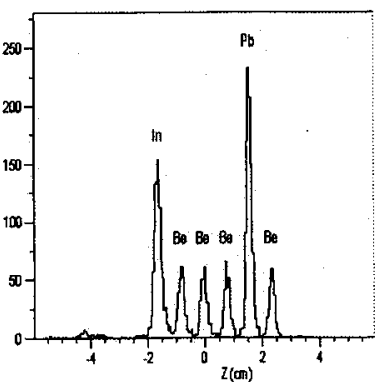

(a)

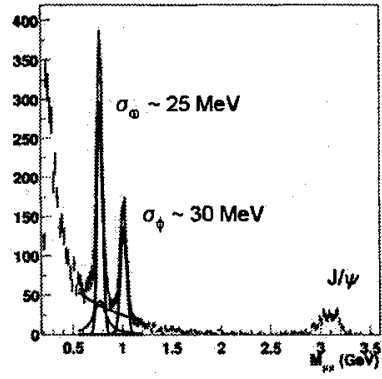

(b)

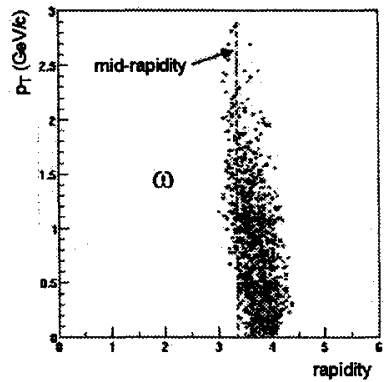

(c)

FIGURE 3. (a) Z-vertex reconstruction capabilities, (b) dimuon mass resolution, and (c) phase space coverage with the microstrip detector telescope in proton-nucleus collisions.

\section{FIRST RESULTS FROM LEAD-LEAD COLLISIONS}

A test run with three completed 4-chip pixel detector planes was performed in October 2002. Low-energy lead beams of 20 and $30 \mathrm{GeV}$ per nucleon were available from the SPS at that time. The run was aimed at a thorough technical test of the detector components under real conditions in the experiment, including the tracking and vertexing algorithms that are used in the on-line and off-line data analysis. The target consisted of three $\mathrm{Pb}$ disks of $1.5,1.0$ and $0.5 \mathrm{~mm}$ thickness. During the run, the pixel detector modules performed very well and yielded efficient particle detection at low noise. Results from the track and vertex reconstruction are shown in Fig. 4. No dipole magnetic field was applied. Already with only three detector planes available, the positions of the vertices on the beam line, both in the targets and the steel windows of the target vacuum box, could be reconstructed with a Z-resolution better than $200 \mu \mathrm{m}$. The vertex resolution in the transverse direction reflects the combined track and vertex finding resolution of the pixel detectors, yielding approximately $20 \mu \mathrm{m}$, which is close to the value that is expected for the full vertex telescope. The correlation width of one of the transverse vertex coordinates from the pixel telescope with the measurement from the beam tracker is approximately $30 \mu \mathrm{m}$. These results confirm that we can reconstruct secondary vertices in heavy ion collisions.
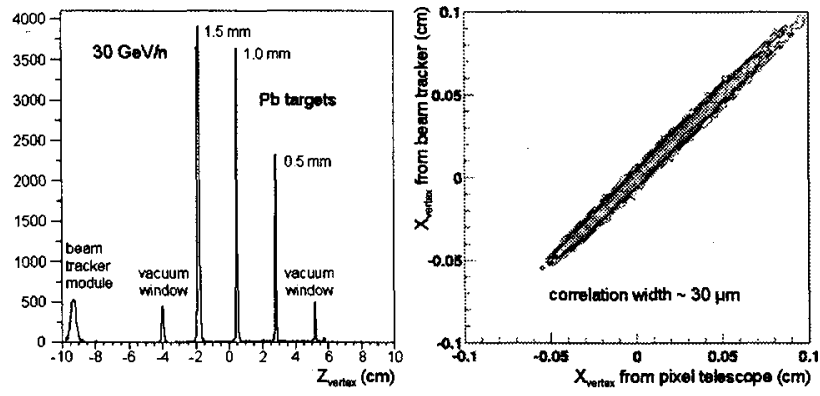

FIGURE 4. Vertex reconstruction performance of three pixel detector planes. 


\section{SUMMARY AND OUTLOOK}

The NA60 collaboration has now fully developed their new detector systems. The completion of the pixel detector telescope for a physics run with heavy ion beams in Fall 2003 is ongoing. High-quality pixel detector components have been delivered from industry. About $75 \%$ of the pixel telescope's 16 tracking planes are already constructed and equipped with their final readout electronics. Further pixel detector planes are presently under construction.

Data collected in 2002 with the NA60 experiment, including a microstrip vertex telescope and a partially installed pixel detector telescope in the vertex spectrometer, confirm its feasibility:

- The dimuon mass resolution at the $\omega$ and $\phi$ mass is approximately $25-30 \mathrm{MeV}$.

- The phase space coverage extends down to low transverse momenta and masses.

- The resolution of the vertex measurement is about $20 \mu \mathrm{m}$ in the transverse coordinates.

In Fall 2003, NA60 will collect data from In-In collisions at $158 \mathrm{GeV}$ per nucleon, to study:

- The patterns of $\mathrm{J} / \psi$ and $\psi^{\prime}$ production to identify the physics mechanism driving charmonium suppression.

- $\rho, \omega$ and $\phi$ production.

- Open charm and thermal dimuon production.

\section{REFERENCES}

1. NA60 Collaboration, Study of prompt dimuon and charm production with proton and heavy ion beams at the SPS, CERN/SPSC 2000-010, March 2000.

2. V. Granata et al., Cryogenic technology for tracking detectors, Nucl. Instr. Meth. Phys. Res. A461 (2001) 197; K. Borer et al., Charge collection efficiency of irradiated silicon detectors operated at cryogenic temperatures, Nucl. Instr. Meth. Phys. Res. A440 (2000)5; P. Rosinský et al., The Cryogenic Silicon Beam Tracker of NA60 for Heavy Ion and Proton Beams, Proc. 11 th Int. Workshop on Vertex Detectors, Hawaii, Nov. 3-8, 2002, submitted to Nucl. Instrum. Methods Phys. Res. A.

3. W. Snoeys et al., Nucl. Instr. Meth. Phys. Res. A465 (2001), 176;

$\mathrm{K}$. Wyllie et al., A pixel readout chip for tracking at ALICE and particle identification at $L H C b$, Proc. Fifth Workshop on Electronics for LHC Experiments, Snowmass, Colorado, 1999. 